\title{
Gastrointestinal bleeding and the prevention of thromboembolism
}

\author{
Corresponding author: \\ Aušra Marcinkonytè, Lithuanian \\ University of Health Sciences, \\ A. Mickevičiaus g. 9, 44307 Kaunas, \\ Lithuania, \\ e-mail: a.marcinkonyte@gmail.com
}

\begin{abstract}
About 2 percent of developed countries population use vitamin $\mathrm{K}$ antagonists (VKA), such as warfarin. These anticoagulation drugs are commonly prescribed for the purpose of thromboembolism prevention, in cases of atrial fibrillation, mechanical heart valves, deep vein thrombosis, pulmonary embolism and etc. The frequency of VKA prescription is massively increasing in the whole world. The main reason for it is the ageing of the population. As the usage of anticoagulants is increasing, doctors more often get to encounter patients with gastrointestinal bleeding taking anticoagulating drugs. The treatment of these patients is difficult because there are several important factors that have to be considered while choosing the tactic of treatment, such as the intensity of bleeding, coagulation, thrombotic risk and endoscopic findings. Even if practical guidelines partially indicate the main principles of actions for similar cases, the treatment of bleeding patients taking anticoagulants remains seriously challenging. This article contains clinical case report and the discussion of the treatment that has been chosen in this case, based on practical guidelines provided by American Society for Gastrointestinal Endoscopy (ASGE), European Society of Gastrointestinal Endoscopy (ESGE) and European Society of Cardiology (ESC). This topic is also addressed in guidelines from the American College of Gastroenterology, the American College of Chest Physicians, the American College of Cardiology, which give similar recommendations.
\end{abstract}

Key words: anticoagulants, warfarin, bleeding, thromboembolism, anticoagulation, endoscopy

Med Res J 2019; 4 (2): 116-119

\section{Introduction}

About 2 per cent of developed countries population use vitamin $\mathrm{K}$ antagonists (VKA), such as warfarin. These anticoagulation drugs are commonly prescribed for the purpose of thromboembolism prevention, in cases of atrial fibrillation, mechanical heart valves, deep vein thrombosis, pulmonary embolism and etc. The frequency of VKA prescription is massively increasing in the whole world. The main reason for it is the ageing of the population. As the usage of anticoagulants is increasing, doctors more often get to encounter patients with gastrointestinal bleeding taking anticoagulating drugs. The treatment of these patients is difficult because there are several important factors that have to be considered while choosing the tactic of treatment, such as the intensity of bleeding, coagulation, thrombotic risk and endoscopic findings. Even if practical guidelines partially indicate the main principles of actions for similar cases, the treatment of bleeding patients taking anticoagulants remains seriously challenging. This article contains clinical case report and the discussion of the treatment that has been chosen in this case, based on practical guidelines provided by American Society for Gastrointestinal Endoscopy (ASGE), European Society of Gastrointestinal Endoscopy (ESGE) and European Society of Cardiology (ESC). This topic is also addressed in guidelines from the American College of Gastroenterology, the American College of Chest Physicians, the American College of Cardiology, which give similar recommendations.

\section{Case report}

52 years old woman having the complaints of vomiting with blood clots, black fieces and general weakness has been delivered to the emergency department.

A day before hospitalization the patient got nausea after coughing, had vomited blood with clots. One day later vomiting occurred once again, black fieces showed up as well. The patient has been delivered to the emergency department by ambulance and has been hospitalized for more detailed examination and treatment.

The patient had rheumatism, mitral valve was replaced with a mechanical prosthesis in 2008. She also 
had a myocardial infarction and coronary arteries bypass in 2008. Artificial cardiac pacemaker (ECS IKDVR) was implanted in 2011. The patient also has arterial hypertension, permanent atrial fibrillation, type II diabetes.

The patient permanently takes metformin, gliclazide, metoprolol, spironolactone, torasemide. She also often takes diclofenac to reduce the pain of her joints. To prevent thromboembolic events which can occur because of mechanical mitral valve prosthesis and permanent atrial fibrillation, the patient takes warfarin.

During the physical examination, the patient was fully conscious, oriented and available for contact. The skin and visible mucous membranes have been pink, the tongue has been dry, covered with white plaque. Breathing rate has been 16 per minute. Breath sound in the lungs has been vesicular, without crackles on both sides. Heartbeat has been arrhythmic with the sound of a mechanical valve's prosthesis. Arterial blood pressure $110 / 80 \mathrm{mmHg}$, heart rate 150 times per minute. The abdomen has been soft, but sensitive in the whole area. The percussion of kidneys hasn't been painful. There have been no oedemas in her legs.

Blood test results: red blood cells (RBC) 3,27 x 10 $12 / \mathrm{l}$, haemoglobin $(\mathrm{Hb}) 103 \mathrm{~g} / \mathrm{l}$. Coagulation tests: SPA $25 \%$, INR 2,19.

Endoscopy showed a bleeding gastric ulcer in the angular area, which has been around $0.7 \mathrm{~cm}$ in size and with a clot underneath. The bleeding has been stopped by clamping. The treatment has been continued with esomeprazole/pantoprazole $80 \mathrm{mg}$ bolus intravenously, continuing $8 \mathrm{mg}$ per hour by automatic syringe pump for 3 days.

A blood test was repeated after the endoscopy and it showed that the amount of haemoglobin was getting lower $(100 \bullet 88 \bullet 81 \mathrm{~g} / \mathrm{l})$. The patient had a blood transfusion, 2 units of red blood cells mass were given.

Next morning the health state of the patient was improved, general weakness seemed to be reduced, there were no signs of bleeding.

The treatment has been continued with Sol. Esomeprazole $40 \mathrm{mg}$ twice a day intravenously for 2 days, later Caps. Omeprazole $20 \mathrm{mg}$ twice a day orally were prescribed.

In case of gastrointestinal bleeding, the decision to stop taking warfarin and change it with low molecular weight heparin $(\mathrm{LMWH})$ was made, so the patient has started getting Sol. Nadroparin 2850 IU twice a day subcutaneously.

Tab. Tardyferon $80 \mathrm{mg}$ once a day orally has been prescribed additionally to treat anaemia.

The patient was discharged from hospital after a week. On the day of discharge, her health was improved, there were no signs of recurrent bleeding. Heartbeat has been arrhythmic with the sound of a mechanical valve's prosthesis. Arterial blood pressure $90 / 64 \mathrm{mmHg}$, heart rate 64 times per minute. Breath sound in the lungs has been vesicular, without crackles on both sides. The abdomen has been soft, slightly sensitive in the epigastric area.

It has been recommended to continue the treatment with Caps. Omeprazole 20 mg twice a day orally, Tab. Tardyferon $80 \mathrm{mg}$ once a day orally, Sol. Nadroparin 2850 IU twice a day subcutaneously. Also to avoid nonsteroidal anti-inflammatory drugs (NSAID), to use Caps. Tramadol if severe pain occurs. It has been planned to repeat endoscopy after a month and then decide if warfarin using should be restarted.

Endoscopy was repeated after a month, the healed ulcer was observed and nadroparin was changed with warfarin again. Warfarin resumption was started after more than a month since gastrointestinal bleeding occurred. There has been found Helicobacter pylori infection and it has been treated with antibiotics. It was also recommended to continue taking iron preparation to treat and prevent anaemia.

\section{Discussion}

The patient is 52 years old woman with the signs of gastrointestinal bleeding- vomiting with blood clots and melena. The patient also uses warfarin, because she has mechanical mitral valve prosthesis and permanent atrial fibrillation, which are dangerous because of increased risk of thromboembolism. Anticoagulant warfarin certainly contributes to avoiding thromboembolic events, yet significantly increases the risk of bleeding. Gastrointestinal bleeding that occurred for the patient might be the result of warfarin usage. Based on literature sources, in cases of active and heavy bleeding, the usage of anticoagulants should be discontinued, except minimal rectal bleeding. [1, 2] In case of this specific patient, bleeding has been active, with a decreasing amount of haemoglobin, consequently, warfarin has been discontinued, according to the recommendations of literature sources.

Early endoscopic intervention is the main measure to stop gastrointestinal bleeding, so this procedure should be organized immediately. [2] Anticoagulants users' blood coagulation, which is mainly reflected by the international normalized ratio (INR), can be over the therapeutic value, that is why it is important to measure INR during the patient preparation for the endoscopic procedure and to take additional means for restoring normal coagulation. According to ASGE practical guidelines, in cases when bleeding is active, but INR value fits in therapeutic range $(<2.5)$, it is not recommended to postpone endoscopic procedure in the purpose of restoring coagulation. However, if INR is $>3$, there is a need to take actions to normalize coagulation of blood. Vitamin $\mathrm{K}$, prothrombin complex concentrate (PCC) or fresh frozen plasma (FFP) might be a choice in 
this case. For all the patients, except those with minimal rectal bleeding and those with INR $<5$, VKA should be discontinued and administered Vitamin K. A decision to give PCC (or FFP if there is no possibility to use PCC) should be taken depending on patient's bleeding intensity, INR value, the timing of endoscopic procedure and individual risk for thrombosis. PCC should be given to intensively bleeding, critical state patients with persistent or permanent haemodynamic instability, even in those cases when INR value fits the therapeutic range. INR should be measured every 20-30 minutes until the end of the PCC infusion. If INR remains $>1.5$, one more dose of PCC should be given. After 6-8 hours INR should be measured again, and then once a day until the situation stays critical. It is recommended to administer Vitamin K together with PCC to avoid coagulopathy which can occur 12-24 hours after INR normalization when infused coagulation factors are eliminated from an organism (the half-life of warfarin is 20-60 hours, PCC 6-8 hours, FFP 1,5 hours- 2 days). Actively bleeding, haemodynamically stable patients with therapeutic INR value should be given only intravenous Vitamin K, which is enough in cases like these. If INR is over a therapeutic range, PCC or FFP should be considered. Urgent restoring of coagulation is not necessary for minor bleeding that does not cause anaemia. If TNS $>5$ low dose of Vitamin K can be given orally (1-5 mg) or intravenously (1-2.5 mg). [1, 3, 4]

In the described situation, INR value has been in the therapeutic range [2,19]. This INR value does not require additional coagulation recovering actions, such as Vitamin K, PCC or FFP. Those means have not been taken and endoscopy has been arranged immediately. Endoscopy has shown a bleeding gastric ulcer, which has been clamped and bleeding has been stopped. Patients with gastrointestinal bleeding are usually treated with proton pump inhibitors (PPIs). When gastrointestinal bleeding is suspected, intravenous PPIs should be administered empirically (e.g. $80 \mathrm{mg}$ esomeprazole bolus $\mathrm{i} / \mathrm{v}$, continuing $40 \mathrm{mg}$ twice a day intravenously) and continued until the exact location of bleeding is detected. [5] The patient has been treated with esomeprazole/pantoprazole $80 \mathrm{mg}$ bolus intravenously, continuing $8 \mathrm{mg}$ per hour by automatic syringe pump for 3 days. The chosen treatment matches recommendations, though it suggests initiating administration of PPIs as soon as gastrointestinal bleeding is suspected. Later on, treatment was continued by Sol. Esomeprazole $40 \mathrm{mg}$ twice a day intravenously for 2 days and Caps. Omeprazole $20 \mathrm{mg}$ twice a day orally afterwards.

After bleeding is stopped endoscopically, a patient should be carefully monitored in case of recurrent bleeding. If the patient used to take anticoagulating agents, it is important to evaluate thrombotic risk and decide whether taking those drugs has to be restarted. Anticoagulants are usually restarted for patients who are haemodynamically stable and the risk for recurrent bleeding is low (based on Forrest classification). [6]

The timing of resuming anticoagulating agents depends on the specific drug, that patient has been using before. Both ASGE and ESC practical guidelines indicate, that if warfarin has been used, it can be restarted at the same evening of the procedure, only if there are no signs of recurrent bleeding. To reach therapeutic effect might take a few days. [6, 4]

It is recommended to give bridging therapy with heparin for patients who have a high risk of thrombosis. The main point of this therapy is the relatively short half-life of heparin (1.5 hours), which allows discontinuing the usage of preparation immediately if bleeding occurs. Bridging therapy should be started patients who used to take warfarin in these cases:

1. Embolic stroke or systemic embolic event within the previous three months

2. Mechanical mitral valve

3. Mechanical aortic valve and additional stroke risk factors

4. Atrial fibrillation and very high risk of stroke (e.g., $\mathrm{CHADS}_{2}$ score of 5 or 6 , stroke or systemic embolism within the previous 12 weeks, concomitant rheumatic valvular heart disease with mitral stenosis)

5. Venous thromboembolism within the previous three months

6. Recent coronary stenting

7. Previous thromboembolism during interruption of chronic anticoagulation [7]

There are 2 kinds of heparin that can be used for bridging therapy: low molecular weight heparin (LMWH) or unfractionated heparin.

$\mathrm{LMWH}$ is recommended for patients with very high (e.g., rheumatic heart disease, permanent atrial fibrillation with recent embolic stroke, mechanical heart valve) or moderate (e.g., active oncological process) arterial thromboembolic risk. Unfractionated heparin is a better choice in case of renal failure or haemodialysis because it is dosing does not depend on renal function. [7]

Heparins can be given in prophylactic, therapeutic or intermediate doses. Therapeutic doses are the best for patients with a potential source of arterial thromboembolism (e.g., atrial fibrillation, mechanical heart valve) or venous thromboembolism, which occurred within the last month. [7]

Intermediate dosing can be applied for patients with atrial fibrillation or venous thromboembolism, which occurred within the last month, but there is a greater concern of bleeding. [7]

Prophylactic dosing is not applicable for patients with atrial fibrillation, because there is no evidence of its efficiency in this case. Doses like these can be applied 
for patients who had venous thromboembolism within 3-12 month. [7]

Bridging therapy is just a temporary solution, which allows reducing thrombotic risk during the acute, life-threatening period. Observational studies' findings show that resuming Vitamin $\mathrm{K}$ antagonists is the best choice after the acute bleeding period is gone, though the most appropriate timing to do so is not that well studied.

3 clinical studies compared the patients, who resumed Vitamin $\mathrm{K}$ antagonists after gastrointestinal bleeding and those who did not. Resuming of Vitamin $\mathrm{K}$ antagonists has been associated with a significantly lower risk of thromboembolic events and death. Furthermore, the risk of recurrent bleeding has remained similar. [1]

The ideal timing of resuming anticoagulation drugs after gastrointestinal bleeding is poorly studied. Practical guidelines do not specify when to restart using anticoagulants and recommend to do it as soon as cardiovascular complications' risk exceeds the risk of bleeding. One of the studies mentioned earlier divided 653 patients into intervals by the time of resuming anticoagulation after bleeding ( $<7$ days; $15-21$ days; 21-30 days; > 30days). Mortality was lower in those cases, when anticoagulation was resumed $<7$ days, 15-21 days and 21-30 days, compared to those, that resumed coagulation $>30$ days. Patients who started using warfarin after $7 \mathrm{~d}$., had approximately 2 fold higher risk of recurrent bleeding and slightly lower risk of thromboembolism, compared to those, who did it after $>30$ days. The incidence of recurrent bleeding was similar in all groups that resumed anticoagulation after $>7$ days, thus it seems that second week after gastrointestinal bleeding might be the most reasonable timing to restart Vitamin $\mathrm{K}$ antagonists. Nevertheless, patients in this study were not divided based on a specific risk for thrombosis and recurrent bleeding. [1]

The patient in the described case, that has permanent atrial fibrillation and mitral valve prosthesis, uses warfarin for prevention of thromboembolic events. According to literature sources, 3 factors have to be considered, while planning to resume anticoagulation- the risk of recurrent bleeding, specific anticoagulant, that patient used to take before, and thrombotic risk. Endoscopy has shown a bleeding gastric ulcer with a clot underneath, so the risk of recurrent bleeding reaches $10-20 \%$. The prosthetic mitral valve is a factor for high thrombotic risk, moreover, she has permanent atrial fibrillation, which means that there is a need for LMWH therapy. $[8,6]$ The patient has started getting Sol. Nadroparin 2850 IU twice a day subcutaneously.

On the day of discharge from the hospital, the usage of warfarin was still suspended and recommended to continue the treatment with Sol. Nadroparin 2850 IU twice a day subcutaneously and decide whether to restart using warfarin after endoscopy, which was planned after almost a month from the day of discharge. However, available sources show that it is safe to restart warfarin after $>7$ days from gastrointestinal bleeding. Studies have not shown a significantly higher risk of bleeding compared to those cases when warfarin was restarted after a month, so it would be reasonable to restart it earlier than it has been done.

All things considered, the treatment for this patient has been chosen wisely, but based on the latest knowledge, more modern viewpoint on restarting anticoagulating drugs could be applied. Even though gastrointestinal bleeding is a serious life-threatening situation, which requires urgent treatment and careful monitoring, since active bleeding period had passed, more concern should be given to the prevention of thromboembolic events.

\section{References}

1. Radaelli F, Dentali F, Repici A, et al. Management of anticoagulation in patients with acute gastrointestinal bleeding. Dig Liver Dis. 2015; 47(8): 621-627, doi: 10.1016/j.dld.2015.03.029, indexed in Pubmed: 25935464.

2. Gutermann IK, Niggemeier V, Zimmerli LU, et al. Gastrointestinal bleeding and anticoagulant or antiplatelet drugs: systematic search for clinical practice guidelines. Medicine (Baltimore). 2015; 94(1): e377, doi: 10.1097/MD.0000000000000377, indexed in Pubmed: 25569664.

3. Hull DR, Garcia DA. Management of warfarin-associated bleeding or supratherapeutic INR. https://www-uptodate-com.ezproxy.dbazes. Ismuni.It/contents/management-of-warfarin-associated-bleeding-or-supratherapeutic-inr?sectionName=Serious $\% 2$ Flife-threatening $\% 20$ bleeding \& topicRef $=104830$ \&anchor $=$ H744870\& source $=$ see link\#H744870. (Jan 02, 2019).

4. Acosta RD, Abraham NS, Chandrasekhara V, et al. ASGE Standards of Practice Committee. The management of antithrombotic agents for patients undergoing GI endoscopy. Gastrointest Endosc. 2016; 83(1): 3-16, doi: 10.1016/j.gie.2015.09.035, indexed in Pubmed: 26621548.

5. Saltzman JR. Approach to acute upper gastrointestinal bleeding in adults. https://www-uptodate-com.ezproxy.dbazes.Ismuni.It/contents/approach-to-acute-upper-gastrointestinal-bleeding-in-adults?search = practical\%20guidlines\%20for\%20gastrointestinal\%20bleeding\%20and\%20anticoagulation\%20therapy\&source=search_result\&selectedTitle $=5 \sim 150 \&$ usage_type $=$ default\&display_rank $=5 \# \bar{H} 5079756$ (Jan 10, 2019).

6. Kamath PS. Management of anticoagulants in patients undergoing endoscopic procedures. https://mww-uptodate-com.ezproxy.dbazes. Ismuni.lt/contents/management-of-anticoagulants-in-patients-undergoing-endoscopic-procedures? sectionName=URGENT\%20PROCEDURES\&topicRef=2547\&anchor $=H 12 \&$ source $=$ see link\#H15. ( Dec 03, 2018)

7. Douketis JD, Lip G. Perioperative management of patients receiving anticoagulants. https://www-uptodate-com.ezproxy.dbazes. Ismuni.It/contents/perioperative-management-of-patients-receiving-anticoagulants? sectionName=BRIDGING\%20ANTICOAGULATION\&topicRef $=2609$ \&anchor $=$ H2126501\& source $=$ see link\#H2126501. (Sep 24, 2018).

8. Gaasch WH, Konkle BA. Antithrombotic therapy for prosthetic heart valves: Management of bleeding and invasive procedures. https:// www-uptodate-com.ezproxy.dbazes.Ismuni.It/contents/antithrombotic-therapy-for-prosthetic-heart-valves-management-of-bleeding-and-invasive-procedures? sectionName $=$ With\%20risk\%20factors\%20for\%20 thrombosis\&topicRef $=8171$ \&anchor $=$ H919063611\&source $=$ see link\#H919063611. (Aug 15, 2016).

9. Babarskienè MR, Bakšytė G, Baronaitè- Dū, et al. Širdies ligų gydymas (algoritmai ir schemos). 3rd ed. Kaunas: UAB "Kardiologijos projektai.; 2011.

10. Halvorsen S, Storey RF, Rocca B, et al. ESC Working Group on Thrombosis. Management of antithrombotic therapy after bleeding in patients with coronary artery disease and/or atrial fibrillation: expert consensus paper of the European Society of Cardiology Working Group on Thrombosis. Eur Heart J. 2017; 38(19): 1455-1462, doi: 10.1093/eurheartj/ehw454, indexed in Pubmed: 27789570. 